\title{
Innovación para el desarrollo económico del Gremio Artesanal
}

\section{Innovation for the economic development of the Artisanal Guild}

\author{
Katya Maya \\ katyamaya09@hotmail.com \\ Corporación Unificada Nacional \\ Colombia \\ https://orcid.org/0000-0002-8567-9037
}

Recibido: 11 de septiembre del 2018

Aprobado: 13 de noviembre del 2018

\begin{abstract}
RESUMEN
El propósito general fue analizar la innovación para la productividad del gremio artesanal del municipio de Aracataca Departamento del Magdalena. Sustentado bajo los postulados teóricos de Robbins (2008), Blázquez (2009), López (2007) entre otros; Desde el punto de vista cualitativo, con el método etnográfico. Diseño de campo. La información fue recabada mediante técnicas de observación y entrevistas cualitativas. La muestra fue de 3 (tres) informantes del gremio artesanal. En los resultados se hace énfasis a la importancia de la innovación, especialmente cuando se trata del trabajo humano, ellos serán los encargados de traducir en acciones concretas los mensajes que envía el mercado en referencia a los productos existentes y necesidades cambiantes de cada segmento de mercado o grupo de clientes. Se evidencia como la innovación no tiene relación únicamente con el ámbito tecnológico, sino que es una capacidad explotada de manera individual puesta en desarrollo por un grupo determinado, donde se presenta un proceso capaz de transformar productos o servicios en forma de satisfacer las necesidades del público en general.
\end{abstract}

Descriptores: Innovación; desarrollo económico, gremio artesanal; productividad; servicio.

\begin{abstract}
The general purpose was to analyze the innovation for the productivity of the artisan guild of the municipality of Aracataca, Department of Magdalena. Sustained under the theoretical postulates of Robbins (2008), Blázquez (2009), López (2007) among others; From the qualitative point of view, with the ethnographic method. Field design The information was collected through observation techniques and qualitative interviews. The sample was of 3 (three) informants of the artisan guild. The results emphasize the importance of innovation, especially when it comes to human work, they will be responsible for translating into concrete actions the messages sent by the market in reference to existing products and changing needs of each market segment or group of clients. It is evident how innovation does not only relate to the technological field, but it is
\end{abstract}


an individually exploited capacity put into development by a specific group, where a process capable of transforming products or services in order to satisfy the needs of the public is presented in general.

Descriptors: Innovation; economic development; artisanal guild; productivity; service.

\section{INTRODUCCIÓN}

A nivel mundial la innovación se ha convertido en un elemento determinante para las organizaciones, en ese sentido, la misma debe tener dirección, dado que innovar sin dirección puede ser caótico y contra producente. Es evidente, que si el contexto cambia, evoluciona, la organización o el individuo, también debe comenzar a crecer simultáneamente, de manera que pueda satisfacer las necesidades del sector, de esta forma, se está frente a otra característica importante como lo es la capacidad de cambio, considerando que cada vez es mayor además de necesario desarrollarse, mejorar y mantener la competitividad.

Desde esta perspectiva, se comprueba que para poner en práctica el proceso de innovación no es primordial la utilización de tecnología, ya que este necesita la implementación de factores internos y externos de la empresa. Es por ello, que esta debe identificar cuáles son los factores internos que posee para poder llevar a cabo este proceso, y estando preparada a aquellos factores externos que le puedan afectar.

Por otra parte, es importante mencionar que la artesanía es un sector con alto potencial en términos de generación de ingresos para el campo, más aun para sectores desmovilizados y víctimas de la violencia. Es importante mencionar que, la base de los productos artesanales, son productos naturales que se encuentran al alcance de la mano, como: arcilla, bambú, cuero, fibras, fique, guadua, lana, madera, tagua, totumo, metales preciosos, entre otros.

Además se considera que el éxito de todo esto dependerá en buena parte del apoyo que el estado le brinde al sector, especialmente de la mentalidad y actitud empresarial que desarrollen los artesanos para lograr el propósito de innovar en productos competitivos de calidad, buen precio y con la capacidad de ofertar un buen volumen de 
productos, buscando con ello, fortalecerse, darse a conocer a nivel nacional e internacional, impulsando y explotado de la mejor forma su talento innato.

Puede señalarse entonces, que la artesanía inclusive, contribuiría a con la formación de la nueva generación, alejándolos del ocio, permitiendo que posean un oficio que además pueda preservar su identidad cultural, prevenir y contrarrestar actitudes violentas, adicciones en los jóvenes, creando espacios de convivencia que conlleven a logro de la paz que se anhela.

\section{DESARROLLO TEÓRICO}

A los alcances de un mayor conocimiento del presente artículo, se desplegó un resumen del trabajo, que constituye su sustentación teórica y que se estructuró de la manera seguida:

\section{Aspectos teóricos referenciales sobre innovación}

Sobre el particular, Robbins (2008) identifica el término innovación como una idea nueva aplicada para iniciar o mejorar un producto, proceso o servicio. La innovación forma parte de una clase especial de cambio, la cual se pone en práctica para cambiar o mejorar la apariencia de algún producto o servicio buscando satisfacer las necesidades de sus clientes.

Por otro lado, Blázquez (2009) señala que la innovación es la capacidad organizativa para convertir una buena idea en un producto, servicio, proceso. Ambos autores afirman que la innovación es una idea que forma parte de la iniciación o mejoramiento de un producto o servicio dado. En este mismo orden de ideas, López (2007) manifiesta que la innovación constituye un proceso de "destrucción creadora" cuyo resultado es el desarrollo económico y empresarial. Indica que es un proceso complejo en el que intervienen personas, equipos físicos y conocimiento.

Es preciso destacar, la importancia de la intervención del recurso humano en los procesos de innovación ya que serán estos los encargados de traducir en acciones concretas los mensajes que envía el mercado en referencia a los productos existentes y a las necesidades cambiantes de cada segmento de mercado o grupo de clientes. Sin embargo, 
Conrado (2006 p. 84) en su concepto refiere lo siguiente: “la innovación es la encarnación, combinación o síntesis del conocimiento en productos, procesos o servicios originales, relevantes y valiosos". También se suele entender este término con la introducción de un objeto o método nuevo.

Al ser considerado como un método nuevo, es de vital importancia considerar el elemento capacitación dado que al desarrollar nuevos productos o servicios puede ser necesario capacitar al personal encargado de presentarlo al cliente para que conozca con detalle los atributos y características del nuevo producto o servicio.

De las definiciones anteriormente expuestas, se evidencia como el término innovación no tiene relación únicamente con el ámbito tecnológico como es relacionado comúnmente. En otras palabras, la innovación es una capacidad explotada de manera individual que es puesta en desarrollo por un grupo determinado, en el cual se presenta un proceso capaz de transformar productos o servicios en una nueva forma de satisfacer las necesidades del público en general.

\section{Capacidad de innovación}

Para, García (2009) la capacidad de innovación es muy valiosa para una empresa, ya que permite responder a las amenazas del entorno actual y aprovechar sus oportunidades. Además, que como recurso intangible, a diferencia de los recursos físicos y financieros, puede ser utilizada en más de una parte de la empresa sin que ello suponga una disminución de su valor en otras áreas.

De esta forma, la utilización de esta capacidad le permitirá a la empresa poseer un activo intangible de gran importancia el cual podrá ser empleado en todas las partes de la organización pudiendo lograr la mejora continua de la misma. Por otro lado, también permite responder de manera más eficaz a las amenazas y oportunidades que se presenten en el entorno de la misma.

En este mismo orden de ideas, Santos (2009) menciona que la capacidad de innovación es una habilidad que se tiene para generar nuevos conocimientos sobre el anterior conocimiento. Esta habilidad es fundamental para la renovación de la empresa y es clave para la creación de éxito sostenible. En otras palabras, esta capacidad permite estar en 
constante generación de cocimientos e ideas nuevas orientadas a satisfacer las necesidades actuales de sus clientes.

En otras palabras, la capacidad de innovación implica aplicar ideas que son nuevas para la empresa y que crean valor superior al cliente, y por ello a la organización. De las definiciones antes expuestas se puede evidenciar que dichos autores coinciden en que es una habilidad que desarrolla el individuo, siendo esta uno de los activos intangibles más importantes. Es por ello, que incorporar el desarrollo de esta habilidad le permitirá a la empresa desarrollar grandes ventajas competitivas que la pueden ayudar a ganar mercado, también puede ser utilizada en distintas áreas de la empresa garantizarle el éxito sostenido a la misma.

Esta gran capacidad fomenta la obtención de mejores resultados dado que el mercado y el entorno son dinámicos, y la innovación permite ser cambiante y dinámico a la vez estando a la par del mercado así lo indica Brenes (2011). Agrega que se considera una capacidad intelectual para explotar nuevas oportunidades de mercado, además de explotar las fuerzas actuales en la estrategia de la empresa. Es por ello, que quien decida invertir en explotar estas capacidades en sus empleados podrán tener una ventaja competitiva mayor que los demás competidores, ya que tiene a su favor el ser dinámico a los cambios que presente el mercado.

\section{Fuentes de innovación}

Según Brenes (2011), debido a la magnitud de la importancia de innovar en el ámbito empresarial, se torna lógico desarrollar y mencionar los aspectos por los cuales se toma esa decisión, la cual a primera vista suena arriesgada pero que en su ejecución termina siendo esencial. De esta manera Santos (2009), agrega que en muchos casos es necesario implementar fuentes de innovación, con indicadores que permitan llegar al cliente, en ciertos casos inclusive sin su petición o consentimiento.

Por otra parte, Deming (2007) mencionan que un cliente repetitivo le deja a la empresa diez veces más beneficios financieros que un cliente impactado por campañas publicitarias, lo cual indica la importancia de ese paso que logre el producto indicado, el servicio deseado o perfecto para el segmento de mercado pretendido. Para, Pages (2008), el punto de partida 
de una organización, para el desarrollo de sus actividades, se da, tomando en cuenta la magnitud de la importancia del producto para la empresa.

Al relacionar las teorías de los autores antes mencionados, se puede unificar criterios que favorezcan al gremio artesanal, en cuanto a tomar en cuenta aspectos significativos para el desarrollo y la aplicación de la innovación en sus operaciones, en este sentido, se mencionan factores importantes de innovación, que al ser tomados en cuenta fortalecerán el sector para la cual se desarrolla la investigación, a saber:

a) Innovación orientada por las necesidades percibidas de los clientes: "Sin jardín no hay jardinero" expresión tomada de Pages (2008) valorada debido a que expresa claramente el punto desarrollado a continuación, donde explica "que sin clientes no hay empresa, sin el estudio específico de sus necesidades no es posible lograr la excelencia", partiendo desde ese punto torna distintos dilemas, en donde se pregunta cuál es la interrogante correcta: ¿A qué cliente le puede servir este producto? o ¿Quién quiero que sea mi cliente?

Este punto explica lo esencial del estudio de mercado al que desea llegar para innovar, para mejorar o para cambiar un producto o servicio. Esta investigación es indispensable ya que si un negocio no está ubicado en el mercado correcto, sirviendo al cliente elegido, con productos y servicios diseñados específicamente para cumplir con sus necesidades y deseos, esta empresa está destinada al fracaso, ahora al hablar de necesidades percibidas el autor habla de las necesidades que el cliente sabe que tiene y que el negocio está obligado a estudiar, tomar decisiones y en base a eso innovar.

b) Innovación orientada por las necesidades no percibidas de los clientes: Si supone interesante estudiar las necesidades percibidas de los clientes, pues estudiar las no percibidas puede ser aún más prometedor y necesario. Un cliente no es experto en materia de innovación, muchas veces ignorante en el tema, por lo que las organizaciones están totalmente obligadas a ampliar sus estudios más allá de lo que el cliente exige.

Donde, Pages (2008) la menciona como "esa información indirecta que se adquiere de estudios de mercado como pueden ser: ¿Dónde vive?, ¿Cómo se comporta? O hasta ¿Cómo compra? Entre otras" que para autores como Valdez (2007) son válidas para establecer el ciclo y estilo de vida de los clientes junto con su objetivo, hasta llegar al punto de empresas decididas a convivir con el cliente para comprenderlos mejor. 
Tomando en cuenta los argumentos que se han presentado esta manera de iniciar en el arriesgado mundo de la innovación tomados de Valdez (2007), recalca que la innovación orientadas en las necesidades no percibidas por el cliente es igual o más importante que la anterior mencionada, necesita un estudio más profundo, donde la información recopilada incluye no solo un análisis enfocado en el cliente que permita especificar no solo lo que este desee, sino lo que no sabe que desea.

Complementando el párrafo anterior es acertado mencionar a un autor que menciona la transcendencia de la aceptación del cliente al comienzo de la innovación; Landrum (2007) señala que el éxito de cualquier impulso creador debe ser la aceptación del mercado, no la invención de sí mismo. Como bien es expresado por los autores, la innovación orientada a las necesidades no percibida por los clientes, debe mantener una relación con el mismo, que permita sin su consentimiento conocerlo, sobretodo entenderlo, y de esta manera evaluar aquellos factores que permitan su satisfacción.

c) Innovación orientada a las necesidades futuras de los clientes: En el inmenso mundo de la mejora continua surgen aspectos circunstanciales que podrían parecer imposibles pero realmente se convierten en claves, ahí surge este nuevo punto donde es posible conseguir una fuente de innovación donde el cliente no ha percibido sus necesidades, pero podría necesitarlas en un futuro. Según, Valdez (2007), hay productos y servicios que el cliente va a necesitar en el futuro y ni siquiera se imagina que puedan llegar a existir. Es más, las empresas que las producirán y venderán tampoco lo saben.

Por otro lado, Gunding (2005) expresa que la innovación debe ir enfocada, a aquellas necesidades, presentadas por el cliente aun sin su consentimiento. Parece increíble y hasta llegar al punto de ilógico la necesidad de evaluar posibles necesidades futuras del cliente, lo cual gracias a la investigación nos permite mencionar que es fundamental es las aspiraciones futuras de la empresa, en su planificación estratégica, sus puntos a mejorar entre otras, es totalmente necesario el conocimiento de las necesidades futuras.

\section{RUTA METODOLOGÍCA}

Desde una perspectiva epistemológica, la investigación cualitativa se orienta hacia la construcción de conocimiento acerca de la realidad social y cultural a partir de la interpretación de la perspectiva de los sujetos involucrados. Metodológicamente, tal 
postura implica asumir un carácter dialógico en la creencia, las mentalidades, los mitos, perjuicios y sentimientos, todos los cuales son aceptados como elemento de análisis para producir conocimiento sobre la realidad humana.

En efecto, como lo señala Rojas (2007) problemas tales como descubrir el sentido, la lógica y la dinámica de las acciones concretas, se convierten en una constante de las diversas perspectivas cualitativas. Desde lo ontológico, el manejo de una concepción de la realidad o de algunos de sus componentes es imprescindible para construir o asumir definiciones, al pensar en la realidad implica transformar en conjunción, entre la reflexión y la acción, desde el conocimiento pleno de nuestros. Desde el punto de vista cualitativo, la investigación se desarrolla con el método etnográfico, el cual es definido por Hernández, Fernández y Baptista (2014, p.700), como el estudio que pretende investigar grupos que comparten una cultura, para lo cual es el investigador quien selecciona el lugar, realiza la detección de sus informantes para luego recolectar y analizar los datos obtenidos, esto se traduce, que la importancia de que este tipo de estudio está en describir, y analizar ideas, significados, conocimientos, de un grupo selecto de individuos, que el caso concreto del presente estudio es el gremio artesanal en el municipio de Aracataca, Departamento del Magdalena.

En correspondencia con lo anterior, está investigación considera el propósito de los estudios etnográficos, por cuanto describe y analiza el fenómeno que se desarrolla la Innovación en el gremio artesanal en el municipio de Aracataca, con respecto analizar la innovación para el desarrollo económico del gremio artesanal del municipio de Aracataca Departamento del Magdalena.

Por lo que los informantes clave que se eligen pueden emitir su opinión de acuerdo a este planteamiento, para luego dejar en los resultados reflejadas las situaciones que se desarrollan, de manera que se puedan emitir reflexiones finales y recomendaciones, cumpliendo con los propósitos del estudio, y finalmente analizar la innovación para la productividad del gremio artesanal del municipio de Aracataca 
Departamento del Magdalena. El cual se convierta en un aporte del estudio al progreso de la investigación científica.

Profundizando, dentro de los estudios etnográficos existen diferentes clasificaciones, tal como lo explica Creswell (2005), esta investigación posee diseño clásico, puesto se trata un estudio típicamente cualitativo, en el cual se analizan temas culturales y las categorías que se vislumbran son inducidas durante el trabajo de campo, asimismo el contexto de la investigación puede ser un grupo de personas que comparten una cultura determinada, practicas e ideas, posiciones ideológicas, valores y mantienen interacciones.

Es propicio considerar, que la etnografía demanda lapsos de tiempo prolongados con los individuaos a ser estudiados, usualmente se utiliza como técnica fundamental la observación, lo que ayuda a estudiar los significados del comportamiento, el lenguaje y las interacciones del grupo con una situación en común, particularmente para esta investigación la transversalidad en las estrategias de enseñanzas en proyectos educativos.

Como método de investigación cualitativo, la observación participante tiene elementos en común con las entrevistas en profundidad. Durante el periodo inicial, la recolección de datos, se deja en un segundo plano, siendo prioritario el conocimiento de las personas y del lugar donde se va a realizar la investigación. Se puede decir que la investigadora que utiliza cualquiera de las dos técnicas debe ir lentamente al comienzo, formulando preguntas no directivas, estableciendo rapport (sintonía psicológica y emocional) y adaptándose a los participantes, antes que preocuparse de los objetivos de la investigación.

Sin embargo, la diferencia más importante entre ambas técnicas se refiere al contexto y situación donde se realizan. Mientas que los observadores participantes llevan a cabo sus estudios en el medio natural, es decir en el lugar donde se encuentran las personas observadas, las entrevistas se hacen en lugares especialmente preparados para ese fin. 
La adaptación de las técnicas de entrevistas, surgirá en el lugar donde se acuerde la cita, no se llevaran preguntas formuladas, como se puede comprender, se iniciará un dialogo, donde se le informará al entrevistado sobre que se estaba investigando, se permitirá abordar la entrevista desde su experiencia, donde surgirán preguntas por la entrevistadora consideradas importantes para investigación.

Se permitirá que el entrevistado aborde temas personales, pero sin perder el propósito de la investigación, habilidad que tiene que tener el investigador de no perder el control de lo que se quiere. En este sentido se tomaran notas de puntos clave de la entrevista, donde los datos obtenidos, serán de exactitud de lo que allí se expresa y que luego ayudaran a la entrevistadora a construir el momento de la entrevista, para luego interpretarlo.

El análisis de contenido, según Bardin (1986) citado por López (2012) es una técnica de investigación para la descripción objetiva, sistemática y cuantitativa del contenido manifiesto de las comunicaciones, que tienen como primer objetivo interpretar. Así, corresponde al campo de análisis de contenido de actividades en las que partiendo de un conjunto de técnicas parciales pero complementarias, consiste en explicar y sistematizar el contenido de los mensajes y la expresión de ese contenido, con la ayuda de los indicios cuantificables, o no.

Todo ello con la finalidad de efectuar deducciones lógicas y justificables concernientes a la fuente (el emisor y su contexto) o eventualmente a los efectos de los mensajes tomados en consideración. Como señala este autor, a pesar que a veces no lo parece el análisis de contenido es un método muy empírico, depende del tipo de discurso en el que se centre y del tipo de interpretación que se persiga, sin embargo no le faltan razón cuando afirma que realmente el análisis de contenido.

No existen plantillas ya confeccionadas y listas para ser usadas, simplemente se cuenta con patrones base, a veces difícilmente traspasable, salvos para usos simples y generalizados, como es el caso de la eliminación próxima a la decodificación de respuestas en preguntas abiertas de cuestionarios cuyo 
contenidos se liquida rápidamente por temas, la técnica del análisis de contenido adecuada al campo y al objetivo perseguido, es necesaria inventarla.

La confiabilidad y validez según Martinez (2010) son procesos claves en los paradigmas cualitativos, donde se establecen supuestos precisos acerca de la comprensión de los fenómenos en una realidad determinada y real donde el investigador no intenta manipular el fenómeno de interés .Sin embargo, la investigación cualitativa por sí es de carácter hermenéutico, tal proceso se entrecruzan en la dinámica dialéctica-interpretativa, donde la investigadora junto al equipo de trabajo están llamados a adquirir una visión amplia y total sobre el fenómeno específico de la realidad estudiada.

Para ello, señala Hidalgo, (2015) el investigador necesita ser creativo, flexible, comunicativo y negociador que construye su propia realidad a través de la experiencia y del diálogo de quienes participan en el proceso. Este proceso propicia la participación de todos sus actores haciendo uso de herramientas como la autoevaluación, coevaluación y heteroevaluación, los cuales permitirán al equipo contrastar la realidad observada desde diferentes ángulos y así concebir significados individuales, de pares y colectivos.

Partiendo de la visión de García (2012), la confiabilidad de perspectiva hermenéutica, se fundamenta en la intuición, la interpretación y el entendimiento. La transversalización entre estos pilares, permiten que la confiabilidad desarrolle un proceso de construcción de la evaluación, sin pretender inducir generalizaciones sino explicar lo particular.

Así, con la confiabilidad hermenéutica, se describe cómo alcanzar la comprensión, la interpretación, los acuerdos y convenimientos, señalando el espacio, tiempo, estrategias para desarrollar el diálogo y la conversación, mecanismos de concertación y cualquier otro elemento que permita tener una visión clara y diáfana del proceso de discusión y conclusión sobre la evaluación de la investigación. 


\section{A MANERA DE REFLEXIONES FINALES}

Es preciso destacar, luego de tomar en cuenta los aciertos, la importancia de la innovación, especialmente cuando se trata del trabajo humano, ya que serán estos los encargados de traducir en acciones concretas los mensajes que envía el mercado en referencia a los productos existentes y a las necesidades cambiantes de cada segmento de mercado o grupo de clientes. Sin embargo, se puede decir, que la innovación es la encarnación, combinación o síntesis del conocimiento en productos, procesos o servicios originales, relevantes y valiosos; también se suele entender como la introducción de un objeto o método nuevo, en este caso, el que los artesanos pueden introducir al mercado.

Al ser considerado como un método nuevo, es de vital importancia considerar el elemento capacitación dado que al desarrollar nuevos productos o servicios, especialmente los que son elaborados como piezas únicas hechas a mano, puede ser necesario capacitar al personal encargado de presentarlo al cliente para que conozca con detalle los atributos y características del nuevo producto o servicio.

Lo antes expuesto, evidencia como la innovación no tienen relación únicamente con el ámbito tecnológico, sino que es una capacidad explotada de manera individual que es puesta en desarrollo por un grupo determinado, en el cual se presenta un proceso capaz de transformar productos o servicios en una nueva forma de satisfacer las necesidades del público en general.

Otra de las situaciones a considerar es que, la utilización de la capacidad de innovar, le permitirá al artesano poseer un activo intangible de gran importancia el cual podrá ser empleado en todas las partes de su trabajo pudiendo lograr la mejora continua del mismo. Por otro lado, también permite responder de manera más eficaz a las amenazas y oportunidades que se presenten en el entorno.

Esta habilidad es fundamental para la renovación de su trabajo y es clave para la creación de un éxito sostenible. En otras palabras, esta capacidad permite estar en constante generación de conocimientos e ideas nuevas orientadas a satisfacer las 
necesidades actuales de sus clientes, sin dejar de considerar lo que es potencialmente comercializable.

Esto es que, la capacidad de innovación implica aplicar ideas que son nuevas y que crean valor superior al cliente. De lo anterior, se puede evidenciar que esta capacidad es una habilidad que desarrolla el individuo, siendo esta uno de los activos intangibles más importantes. Es por ello, que incorporar el desarrollo de esta habilidad le permitirá desarrollar grandes ventajas competitivas que la pueden ayudar a ganar mercado, también puede ser utilizada en distintas áreas para garantizarle el éxito sostenido.

Por tanto entonces, a nivel mundial la innovación se ha convertido en un elemento determinante para las organizaciones, en ese sentido, la misma debe tener dirección, dado que innovar sin dirección puede ser caótico y contra producente. Es evidente, que si el contexto cambia, evoluciona, la organización o el individuo, también debe comenzar a crecer simultáneamente, de manera que pueda satisfacer las necesidades del sector, de esta forma, se está frente a otra característica importante como lo es la capacidad de cambio, considerando que cada vez es mayor además de necesario desarrollarse, mejorar y mantener la competitividad.

Desde esta perspectiva, se comprueba que para poner en práctica el proceso de innovación no es primordial la utilización de tecnología, ya que este necesita la implementación de factores internos y externos de la empresa. Es por ello, que esta debe identificar cuáles son los factores internos que posee para poder llevar a cabo este proceso, y estando preparada a aquellos factores externos que le puedan afectar. Por otra parte, es importante mencionar que la artesanía es un sector con alto potencial en términos de generación de ingresos para el campo, más aun para sectores desmovilizados y víctimas de la violencia. Es importante mencionar que, la base de los productos artesanales, son productos naturales que se encuentran al alcance de la mano, como: arcilla, bambú, cuero, fibras, fique, guadua, lana, madera, tagua, totumo, metales preciosos, entre otros.

Además se considera que el éxito de todo esto dependerá en buena parte del apoyo que el estado le brinde al sector, especialmente de la mentalidad y actitud empresarial 
que desarrollen los artesanos para lograr el propósito de innovar en productos competitivos de calidad, buen precio y con la capacidad de ofertar un buen volumen de productos, buscando con ello, fortalecerse, darse a conocer a nivel nacional e internacional, impulsando y explotado de la mejor forma su talento innato. Así mismo es importante destacar lo señalado por Zambrano (2018) sobre el papel de las universidades en el acompañamiento de empresas de propiedad social, la autora expresa que:

la vinculación real de la Universidad con el entorno productivo y comunal de la región, se ha desarrollado de manera práctica en el municipio Iribarren, debido a que es en esta localidad donde se encuentra directamente inmersa la universidad, además de ser la zona más poblada de todo el Estado Lara, constituye la parte central de la capital del estado, y es donde se está dando el cambio de gestión en algunas empresas a nivel industrial, en rubros de extrema importancia para todo el país como lo son el alimenticio y el de construcción (p. 55).

Lo anteriormente planteado permite inferir la importancia de la territorialización de las universidades como parte de las políticas pública del Estado que permitan garantizar el acompañamiento productivo de este importante sector.

\section{REFERENCIAS CONSULTADAS}

1. Blázquez, M. (2009). Desempeño Organizacional: Mejora, Creación e Incubación de nuevas organizaciones. Global Business Press. Bloomington, Indiana, Estados Unidos.

2. Brenes, C. (2011). Comunidades rurales. Criterios y herramientas para su diagnóstico. Editorial Universidad Estadal a Distancia (EUNED). Primera Edición, San José, Costa Rica.

3. Conrado Arias, Miró. (2007). Innovación, modelo de dirección para la innovación MDI. (1ra. Ed). España. Mayo (2007). Colección EOI.

4. Creswell, J. (2005). Qualitive inquiry and research design. Sage Publicaciones. Londres.

5. Deming (2007). Cuaderno de Innovación: Una iniciativa para la implantación de la Calidad. 
6. García, H. (2009). Política e innovación tecnológica: perspectivas económicas. Monte Ávila Editores. Colombia.

7. Gunding (2005) Desarrollo de la innovación en el mundo. Disponible en: www.redalyc.org/pdf/340/34004507.pdf.

8. Hidalgo, L (2015) Confiabilidad y Validez en el Contexto de la Investigación y Evaluación Cualitativas. Universidad Central de Venezuela. Caracas Venezuela.

9. Landrum (2007) Desarrollo de la innovación en el mundo. Disponible en: https://www.scribd.com/.../Gestio-n-de-la-innovacio-n-desde-la-dimensio-nhumana.

10.López, E. (2007). La innovación continua en el éxito empresarial. Universidad Nacional de Educación a Distancia Madrid. España.

11. Martínez, A. (2010). Los Conceptos de Conocimiento, Epistemología y Paradigma, como Base Diferencial en la Orientación Metodológica del Trabajo de Grado.

12.Pages, C. (2008). Protocolo Interempresarial. Ediciones INNOVA. Málaga, España.

13. Robbins, S (2008). Administración. Octava Edición. Pearson Educación. México.

14.Rojas, V. (2007). Competencias en la comunicación: Hacia las prácticas del discurso. ECOE Ediciones. Tercera Edición. Bogotá, Colombia.

15.Santos, M. J. (2009). Innovación Tecnológica y Procesos Culturales. Fondo De Cultura Económica Us.

16. Valdéz, L. (2007) La re-evolución empresarial del siglo XXI. Bogotá: Editorial Norma.

17.Zambrano Durán, J. (2018). EL ACOMPAÑAMIENTO DE LA UNIVERSIDAD A LAS EMPRESAS DE PROPIEDAD SOCIAL DIRECTA COMUNAL: MODELO DE TRANSFORMACIÓN UNIVERSITARIA. Revista Arbitrada Interdisciplinaria Koinonía, 2(4), 43-59. Recuperado de http://fundacionkoinonia.com.ve/ojs/index.php/revistakoinonia/article/view/69/56.

(C2019 por el autor. Este artículo es de acceso abierto y distribuido según los términos y condiciones de la licencia Creative Commons Attribution (http://creativecommons.org/licenses/by/4.0/). 